\title{
Older Persons "Lost" to the COVID-19 Vaccination: Where Are They?
}

\author{
M. Cesari ${ }^{1}$, B. Vellas $^{2}$

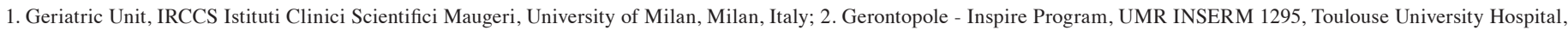 \\ University of Toulouse Paul Sabatier, Toulouse, France

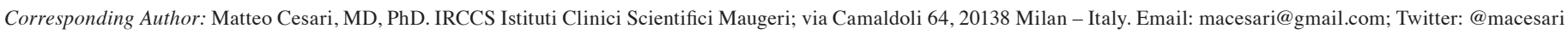

Key words: Vaccines, geriatrics, frailty, integrated care, social care.

T The COVID-19 pandemic has substantially changed our lives. It has also acted as a sort of stress test for care systems, letting emerge all the inconsistencies, weaknesses, and contradictions of them. In particular, frail persons have shown to be those paying the most severe consequences of the general disservices (1). To date, in the absence of specific drugs against the SARS-CoV-2, preventive measures against infection and vaccination represent the only available weapons. Social distancing, hand hygiene, and protective personal equipment have been immediately put in place since the very first phases of the pandemic. Starting in December 2020, vaccines against the SARS-CoV2 infection have been made available and mass campaigns of vaccinations have started worldwide.

Given the high-risk profile exhibited by older persons with frailty, these have been usually prioritized in the vaccination campaigns. Today, the vaccine administration is primarily focused to adults and young persons. Whereas it is generally assumed that the older population has now been vaccinated, a recent survey has suggested that many persons aged 80 years and older (almost 20\%) are not yet (2). Indeed, as reported, "statistics from the Centers for Disease Control and Prevention showed this population's vaccination rates soaring through the spring, then hitting a plateau".

Which are the barriers precluding the vaccination of so many persons at risk of the most severe consequences of COVID-19? Several reasons can be hypothesized:

- Older persons may refuse the vaccination for personal opinions or because influenced by their proxies. In this context, the presence of cognitive impairment and difficulties in judgment might affect the capacity to decide, relying on what younger persons (potentially less concerned by the severity of the virus and more exposed to fake news) choose for them. In this context, it is important to consider that the many no-vax messages might have, directly and indirectly, influenced the most vulnerable ones (due to their frail status and/or low socio-cultural conditions).

- The frailty status of many older persons can complicate the access to the vaccination. Difficulties in the use of technologies for scheduling an appointment, mobility impairment and/or social isolation hampering the possibility to reach the vaccination site, cognitive disorders affecting the capacity to take and retain the appointment... are all examples of potential underestimated barriers.

- The pandemic has made clear that the hospital-centered design of our healthcare systems is not suitable for many persons living with frailty (3). Their protection implies the adoption of a more comprehensive approach, leaving the traditional standalone disease-model in favor of a holistic vision of the individual inclusive of his/her environment. In this context, it cannot be ignored how most of the vaccination campaigns are centered on hubs in the community where persons can go to receive their vaccine dose. However, relatively low interest has been put for supporting primary care and facilitate the vaccination of the frailest individuals who are home-bound. Indeed, the COVID-19 pandemic has exposed the extreme paucity of resources and infrastructures devoted to older persons where they live and age (i.e., in the community). More research is needed to better understand how many and why older persons are still "lost" to our care systems. This is pivotal to develop future strategies allowing the provision of preventive care in the community to the most vulnerable persons.

Access to care has been extremely difficult for many persons over the past months, not only because of the restrictions and lockdowns applied by governments during the hardest moments of the pandemic. Older persons have specially suffered the fragmentation of care and the prolonged disruptions of services (often motivated by the need of facing the COVID-19 emergency). The procrastination of routine clinical evaluations, often combined with the older person's fear of being infected, has uphold many interventions that were instead needed (4). Furthermore, the lifestyle modifications forcedly brought by the pandemic have negatively impacted on the health status of the most vulnerable persons, worsening their functions and clinical conditions $(5,6)$. The functional loss and social isolation developed by older persons over the past months will likely result in major consequences in the next future, both in terms of 1) frailer and more complex patients, and 2) incapacity of services to adequately address the increasing demands.

Interestingly, a recent study by Ankuda and colleagues (7) has recently described an exponential increase of community- 
dwelling older persons who have become home-bound (i.e., leaving the house once a week or less) during these months of pandemics. These persons are exposed to particularly high risk of negative outcomes. Their risk profile is further enhanced by their social isolation preventing them from prompt access to care. A further example is coming from Italy. During the vaccination campaign, almost 500-thousand persons (that is about $1 \%$ of the Italian population) were untraceable and difficult to reach. They are socially isolated, tend to live in rural areas, have no internet/phone connection, and/or move frequently across the country. In other words, the COVID-19 pandemic is showing the existence of a population of frail individuals for which a completely different model of care is needed. The usual reactive approach is evidently not working for them, and proactive/preventive strategies are needed.

Under the current COVID-19 situation, we would like to stress the importance of the following points:

1. With the aging of our population and the increasing number of socially isolated individuals, the system cannot anymore just wait for the incoming request. The continuation of this obsolete approach will contribute at accelerating the collapse of the systems which are designed for late interventions. It is necessary to reshape our clinical and public health strategies for anticipating the problems and act when the case is still reversible (for the benefit of the person and the community) (8).

2. Instead of waiting that the problem arrives to the attention of clinical and social services, it is necessary to identify the early signs of future issues to preventively intervene. This means the building of multidisciplinary bridges facilitating the sharing of relevant information across settings for the development of person-centered actions.

3. In this context, it is noteworthy the work conducted by the World Health Organization (WHO) to promote the integration and continuum of care (9). The WHO has repeatedly recommended over the past years to modify the approach to older persons by implementing preventive strategies and personalization of interventions (e.g., ICOPE Program) (10). Every point of contact between the individual and the care system should become an opportunity for estimating the residual reserves (i.e., intrinsic capacity) and abilities (i.e., functional ability) (11). The resulting information may then be used to track his/her trajectories and identify deviations from the normality.
4. Finally, the adoption of shared technologies is not an option anymore. Indeed, in a world dominated by technologies, it is not anymore acceptable that persons are "lost" to the care system. It is time to take advantage of technologies as exemplified by the ICOPE Monitor, an innovative digital healthcare program designed for community-dwelling older persons with frailty with the final aim of remote monitoring their health status (via nurse assistance) and facilitating access to preventive services (including COVID-19 vaccination) $(12,13)$.

\section{References}

1. Merchant R. COVID-19: role of integrated regional health system towards controlling pandemic in the community, intermediate and long-term care. J Frailty Aging. 2020:1-2. doi:10.14283/jfa.2020.39.

2. Span P. More Than 80 Percent of Seniors Are Vaccinated. That's 'Not Safe Enough.' The New York Times. https://www.nytimes.com/2021/09/02/health/covid-vaccinesseniors.html. Published September 2, 2021. Accessed September 3, 2021.

3. Astrone P, Cesari M. Integrated Care and Geriatrics: A Call to Renovation from the COVID-19 Pandemic. J Frailty Aging. October 2020:1-2. doi:10.14283/jfa.2020.59.

4. Briguglio M, Giorgino R, Dell'Osso B, et al. Consequences for the Elderly After COVID-19 Isolation: FEaR (Frail Elderly amid Restrictions). Front Psychol. 2020;11:565052. doi:10.3389/fpsyg.2020.565052.

5. Kirwan R, McCullough D, Butler T, Perez de Heredia F, Davies IG, Stewart C. Sarcopenia during COVID-19 lockdown restrictions: long-term health effects of short-term muscle loss. Geroscience. 2020;42(6):1547-1578. doi:10.1007/s11357020-00272-3.

6. Canevelli M, Valletta M, Toccaceli Blasi M, et al. Facing Dementia During the COVID-19 Outbreak. J Am Geriatr Soc. 2020;68(8):1673-1676. doi:10.1111/ jgs. 16644

7. Ankuda CK, Leff B, Ritchie CS, Siu AL, Ornstein KA. Association of the COVID-19 Pandemic With the Prevalence of Homebound Older Adults in the United States, 2011-2020. JAMA Internal Medicine. August 2021. doi:10.1001/ jamainternmed.2021.4456.

8. Barusch A, Waters D. Social engagement of frail elders. J Frailty Aging. 2012;1(4):189-194

9. World Health Organization, Department of Ageing and Life Course. Integrated Care for Older People.; 2017. http://www.ncbi.nlm.nih.gov/books/NBK488250/. Accessed March 1, 2019

10. Integrated Care for Older People (ICOPE): Guidance for Person-Centred Assessment and Pathways in Primary Care. World Health Organization; 2019.

11. World Health Organization (WHO). Decade of Healthy Ageing.; 2020. https://www. youtube $. \mathrm{com} /$ watch $\mathrm{v}=$ ShmemfpkVLQ\&list=PL1F160112BFDBC1D5\&index $=2$. Accessed April 27, 2021.

12. González-Bautista E, De Souto Barreto P, Virecoulon Giudici K, et al. Frequency of Conditions Associated with Declines in Intrinsic Capacity According to a Screening Tool in the Context of Integrated Care for Older People. J Frailty Aging. August 2020. doi: $10.14283 /$ ja. 2020.42

13. Tavassoli N, Piau A, Berbon C, et al. Framework Implementation of the INSPIRE ICOPE-CARE Program in Collaboration with the World Health Organization (WHO) in the Occitania Region. J Frailty Aging. 2021;10(2):103-109. doi:10.14283/ jfa.2020.26.

How to cite this article: M. Cesari, B. Vellas. Older Persons "Lost" to the COVID-19 Vaccination: Where Are They? J Frailty Aging 2021;10(4)308-309; http://dx.doi. org/10.14283/jfa.2021.37 